\title{
Functional Heterogeneity at Dopamine Release Sites
}

\author{
James A. Daniel, ${ }^{1,2}$ Sally Galbraith, ${ }^{3}$ Lorraine Iacovitti, ${ }^{4}$ Andrea Abdipranoto, ${ }^{1,2}$ and Bryce Vissel ${ }^{1,2}$ \\ ${ }^{1}$ Neuroscience Program, Garvan Institute of Medical Research, Sydney, New South Wales 2010, Australia, ${ }^{2}$ Faculty of Medicine and ${ }^{3}$ School of Mathematics \\ and Statistics, University of New South Wales, Sydney, New South Wales 2052, Australia, and ${ }^{4}$ Department of Neurology, Thomas Jefferson University \\ Medical College, Philadelphia, Pennsylvania 19107
}

\begin{abstract}
Although drugs used to treat several neurological diseases are presumed to target synapses that secrete dopamine (DA), relatively little is known about synaptic vesicle (SV) release mechanisms at single DA synapses. We found that the relative probability of release $(\operatorname{Pr})$ varied between individual DA synapses. Furthermore, DA terminals generally exhibited lower Pr than glutamatergic hippocampal (Hpc) terminals, suggesting that DA release is less reliable than the release of glutamate. Our mathematical model of fluorescence loss shows that $\mathrm{Pr}$ is regulated by two independent and heterogeneous elements. First, the size of the recycling SV pool regulates Pr. Second, Pr is also independently regulated by additional factors, which are reflected in the time constant of FM 1-43 destaining, $\tau$. We found that the observed difference in Pr between Hpc and DA neurons results because the recycling SV pool is smaller in DA neurons than in Hpc neurons. However, $\tau$ does not vary between these two neuron populations. We also identified a population of functional nonsynaptic boutons in DA axons, which are not associated with a postsynaptic element and which are not functionally different from boutons that formed conventional synapses. Our work provides a new approach to the study of SV exocytosis in DA neurons and shows that synaptic terminals of DA neurons are functionally heterogeneous and differ from excitatory terminals in terms of Pr.
\end{abstract}

\section{Introduction}

Neurons that secrete dopamine (DA) are associated with a number of prevalent neurological disorders, including Parkinson's disease (PD). Drugs used in the treatment of PD, as well as antipsychotics and several addictive drugs, are presumed to act by altering the function of DA synapses. Understanding the mechanisms and signaling pathways that regulate $\mathrm{SV}$ exocytosis, endocytosis and recycling in DA neurons is critical for understanding the actions of these drugs and for the identification of new therapies. However, relatively little is known regarding cellular mechanisms that regulate the exocytosis of these synaptic vesicles (SVs) in DA neurons at the level of individual synapses.

Synaptic transmission is an innately unreliable process, in that each action potential does not always result in neurotransmitter release (Hessler et al., 1993; Rosenmund et al., 1993). The pre-

\section{Received March 20, 2009; revised July 7, 2009; accepted 0ct. 7, 2009.}

Our work was supported by the National Health and Medical Research Council Australia Grant \#188819 (B.V.), a NSW State Government Spinal Cord Injury \& Related Neurological Conditions Research Grant administered by the Office for Science and Medical Research (B.V.), The NSW State Government's BioFirst Award (B.V.), the Australian Postgraduate Award (J.A.D.), the Baxter Family Scholarship (J.A.D.), the Gowrie Trust Scholarship (J.A.D.), The Baxter Trust, The Lyndsay and Heather Payne Medical Research Foundation, Perpetual Philanthropic Foundation, National Institutes of Health Grant NS32519-15 (L.I.), Amadeus Energy Ltd., Bill Gruy, Geoff Dixon, and Dawn Dixon, and the Henry H. Roth Charitable Foundation. This work would not have happened without Amadeus Energy Ltd. Bill and Laura Gruy, and Geoffrey Towner. We thank Nick Kell and Joanna Knott for their support, Drs. T. Lewis, M. Christie, and J. Clements for their insightful comments regarding data analysis, and Drs. S. Janmaat and J. Bekkers for their generous overall assistance. Dr. D. Sulzer provided the methods for culturing dopamine neurons. Dr. J. Bekkers provided the methods for culturing hippocampal neurons. We thank Charles F. Stevens for his invaluable advice. We also thank the following people for their helpful comments in the preparation of this manuscript: Dr. S. Janmaat, A. Daniel, Dr. E. Vissel, Dr. D. Ryugo, Dr. R. Callister, and Dr. S. Oleskevich.

Correspondence should be addressed to Bryce Vissel, Neuroscience Program, Level 7, Garvan Institute of Medical

Research, 384 Victoria Street, Darlinghurst, NSW 2010, Australia. E-mail: b.vissel@garvan.org.au.

D0I:10.1523/JNEUROSCI.1349-09.2009

Copyright $\odot 2009$ Society for Neuroscience $\quad 0270-6474 / 09 / 2914670-11 \$ 15.00 / 0$ synaptic probability of release $(\operatorname{Pr})$ refers to the likelihood that an action potential will cause release of neurotransmitter at a given synapse. Pr is heterogeneous across hippocampal (Hpc) synapses (Rosenmund et al., 1993; Murthy et al., 1997; Ryan et al., 1997). However, mechanisms that govern this functional heterogeneity remain elusive. Moreover, while studies using electrochemical and ultrastructural methods have contributed substantially to our understanding of quantal DA release from SVs (Nirenberg et al., 1997; Pothos et al., 1998; Staal et al., 2004), functional heterogeneity has not been investigated in single DA synapses.

Studies using optical techniques to SV release in Hpc neurons have greatly enhanced our understanding of presynaptic function [for examples, see the studies by Murthy et al. (1997), Ryan et al. (1997), Waters and Smith (2002), Virmani et al. (2006), Chen et al. (2008), Tokuoka and Goda (2008)]. In this study, we provide a description of functional heterogeneity across DA synapses and a comparison of SV exocytosis in DA and glutamatergic $\mathrm{Hpc}$ neurons. We find that DA synapses exhibit considerable heterogeneity in Pr. Overall, DA synapses exhibit lower Pr compared to Hpc synapses, suggesting that DA release is even less reliable than the release of glutamate. Furthermore, by modeling our data we find that Pr can be described by two independent factors. Using this analytical approach, we conclude that the lower Pr in DA neurons is due to these synapses containing fewer recycling SVs available for release. We show that while the kinetics of SV pool release is also heterogeneous at DA synapses, there is no detectable difference in release kinetics between $\mathrm{Hpc}$ and DA synapses. Finally, in addition to synaptic terminals, we show that DA neurons possess a subpopulation of nonsynaptic boutons, which appear functionally similar to synaptic terminals of DA neurons. Our work presents a new approach to the study of SV release in DA neurons, with implications for understanding DA secretion 
at the level of single synapses and examining DA synapses in human disease and therapeutics.

\section{Materials and Methods}

All reagents were from Sigma-Aldrich, unless otherwise stated.

Primary glial culture. Pregnant female mice were either imported from the Animal Resources Centre (Perth, Australia) or bred on-site at the Garvan Institute. Glia were prepared from postnatal day 1 mouse pups. Cortical tissue was collected from pups and dissociated in 0.006\% Tryp$\sin$ (in EBSS, Invitrogen) at $37^{\circ} \mathrm{C}$ for $30 \mathrm{~min}$. Tissue was triturated in culture medium consisting of MEM (Invitrogen), $0.1 \%$ Mito + serum extender (BD Biosciences), $2.94 \mathrm{~g} / \mathrm{L}$ glucose (final), and $10 \%$ fetal bovine serum (FBS, ThermoTrace). The cell filtered through a $70 \mu \mathrm{m}$ cell strainer (BD Biosciences) and cultured in $75 \mathrm{~cm}^{2}$ flasks at $37^{\circ} \mathrm{C}, 5 \% \mathrm{CO}_{2}$. After 10-14 d in culture, glial cells were used to seed $25 \mathrm{~mm}$ glass coverslips coated with $0.1 \mathrm{mg} / \mathrm{ml}$ poly-D-lysine and $0.5 \mathrm{mg} / \mathrm{ml}$ rat tail collagen (BD Biosciences).

Primary midbrain/striatal neuron culture. Our method for culturing midbrain neurons was based on a previously published method (Rayport et al., 1992). Midbrain regions from postnatal day 0 (P0) neonatal mice were collected by microdissection. Tissue chunks were dissociated using papain $(20 \mathrm{U} / \mathrm{ml})$ in the presence of $10 \mu \mathrm{g} / \mathrm{ml}$ kynurenic acid. Tissue was triturated and plated at $6 \times 10^{4}$ cells/well in 6 well plates containing 25 $\mathrm{mm}$ coverslips that had a monolayer of established glial cells (see above). Cells were plated in $1 \mathrm{ml}$ of basic medium (MEM w/10\% heat-inactivated iron-supplemented calf serum) and allowed to settle in an incubator $\left(37^{\circ} \mathrm{C}, 5 \% \mathrm{CO}_{2}\right)$ for $1-2 \mathrm{~h}$. Three milliliters of neuronal culture medium was then added, consisting of 46.5\% MEM, 40\% DMEM, 10\% Ham's F12, 0.25\% Albumax II BSA (Invitrogen), 0.5 mM glutamine, $5.86 \mathrm{mg} / \mathrm{ml}$ D-glucose, $6.16 \mu \mathrm{g} / \mathrm{ml}$ catalase (Worthington), $10 \mu \mathrm{g} / \mathrm{ml}$ kynurenic acid, $125 \mathrm{~nm}$ hydrocortisone, $200 \mathrm{~nm}$ progesterone, $25 \mu \mathrm{g} / \mathrm{ml}$ insulin, 100 $\mu \mathrm{g} / \mathrm{ml}$ apo-transferrin, $30 \mathrm{~nm}$ tri-iodothyronine, $30 \mathrm{~nm}$ sodium selenite, $21.4 \mathrm{U} / \mathrm{ml}$ superoxide dismutase, $15 \mu \mathrm{m}$ putrescine, and $1 \%$ heatinactivated iron-supplemented calf serum. Cells were treated at the time of plating with glial-derived neurotrophic factor (GDNF, Alomone) at 10 $\mathrm{ng} \mathrm{ml}^{-1}$ to promote midbrain neuron survival. Midbrain neurons were left for up to $24 \mathrm{~h}$ before striatal cells were added to each well (see below).

As a modification to the method of Rayport et al. (1992), we cocultured midbrain neurons in the presence of primary neurons taken from the striatum, as striatal neurons constitute a major target for DA neurons of the midbrain. To culture striatal neurons, pieces of striatum were dissected from the brains of $\mathrm{P} 0$ neonatal mice. The tissue chunks were then dissociated using papain and triturated as described for midbrain neurons. The striatal cells were then added to culture wells containing midbrain neurons at a density of $1.5 \times 10^{5}$ cells/well. This resulted in a midbrain/striatal neuron coculture. Cells survived without feeding for several weeks, and were left in culture for at least $15 \mathrm{~d}$ before they were used in experiments. For a discussion of our choice of age for our cultures, see supplemental ST1a, available at www.jneurosci.org as supplemental material.

Primary Hpc neuron culture. For culturing Hpc neurons, the dissection of the hippocampal CA1 region was based on a method previously published (Bekkers and Stevens, 1989). The CA1 of P0 neonatal brains were dissected and then dissociated using papain as described above. CA1 neurons were triturated and then plated at a density of $5 \times 10^{4}$ cells/well on coverslips that had an established monolayer of glial cells. Cells were maintained in $3 \mathrm{ml}$ of neuronal culture medium alone (media contents described above). Neurons were fed weekly by exchanging $1 \mathrm{ml}$ of medium in each well. Hpc neurons were used after at least $15 \mathrm{~d}$ in vitro (see supplemental ST1a, available at www.jneurosci. org as supplemental material).

Immunocytochemistry. Cells were first fixed in $4 \%$ paraformaldehyde (with $4 \%$ sucrose, in PBS) for $2 \mathrm{~min}$ at $23^{\circ} \mathrm{C}$, and then in methanol at $-20^{\circ} \mathrm{C}$ for $10 \mathrm{~min}$. Blocking was performed for $30 \mathrm{~min}$ using $10 \%$ bovine serum albumin (BSA, in PBS). Primary antibodies were: mouse antibassoon (1:200, Stressgen), rat anti-DAT (1:1000), guinea pig antiVGLUT1 (1:1000), mouse anti-Map2 (1:500), mouse anti-PSD-95 (1: 500), rabbit anti-VMAT2 (1:1000, Millipore), rabbit anti-Synaptophysin (1:1000, DAKO), mouse anti-Synaptophysin (1:1000), and mouse anti-
VAMP2 (1:500, Synaptic Systems). Primary antibodies were diluted in $3 \%$ BSA and applied overnight at $4^{\circ} \mathrm{C}$ (except anti-VMAT2, which was applied for $48 \mathrm{~h}$ ). Primary antibodies were detected using secondary antibodies conjugated to AlexaFluor 488, 594, 647, or Pacific Blue (Invitrogen). These fluorophores were stimulated using lasers with emission wavelengths of $488,594,633$, and $405 \mathrm{~nm}$, respectively.

Imaging. Cells were imaged in an RC21-BRFS chamber (Warner Instruments) and perfused at $0.5 \mathrm{ml} / \mathrm{min}$. FM 1-43 studies were performed at $23^{\circ} \mathrm{C}$ in Tyrode's buffer $(136 \mathrm{~mm} \mathrm{NaCl}, 2.5 \mathrm{~mm} \mathrm{KCl}, 2 \mathrm{~mm} \mathrm{CaCl} 2,1.3$ $\mathrm{mm} \mathrm{MgCl} 2,10 \mathrm{~mm}$ HEPES, $10 \mathrm{~mm}$ D-glucose, $\mathrm{pH}$ adjusted to 7.35 with $\mathrm{NaOH})$. FM 1-43 (95 $\mu \mathrm{g} / \mathrm{ml}$, Invitrogen) was delivered to cells for $40 \mathrm{~s}$, followed by depolarization for $100 \mathrm{~s}$ using buffer containing FM 1-43 and $90 \mathrm{~mm} \mathrm{~K}{ }^{+}$. This labeling method was chosen because it gave complete loading of the recycling pool of SVs (see Relationship between Pr and FM dye destaining, below). Cells were then perfused with FM 1-43 alone for $90 \mathrm{~s}$ and washed for $15 \mathrm{~min}$ in buffer containing NBQX (10 $\mu \mathrm{M}$, Alexis Biochemicals) and AP-5 (50 $\mu \mathrm{M})$. FM 4-64 (100 $\mu \mathrm{g} / \mathrm{ml}$, Invitrogen) labeling was performed using the same procedure.

Destaining of FM dye was induced by field stimulation using parallel electrodes ( $1 \mathrm{~ms}$ pulses at $10 \mathrm{~V}$, using S48 stimulator, Grass-Telefactor), $60 \mathrm{~s}$ after imaging commenced. FM 1-43 was imaged using the 488 line of an ArKr laser (Leica DMIRE2), while FM 4-64 was imaged using the 514 line. Images were acquired every $5 \mathrm{~s}$. Laser power, line averaging (2 line scans were averaged per frame) and frequency of imaging were optimized to ensure data exhibited low noise while also minimizing photobleaching (which amounted to $\sim 10 \%$ of total fluorescence over 8 min of imaging). Images were acquired using a Leica $63 \times$, numerical aperture 1.5 oilimmersion objective.

After each experiment, we noted the cell's location in a photo-etched grid on the coverslip (Bellco) and cells were fixed. The same neuron was located after immunolabeling and imaged. The image of the immunolabeled neuron was overlaid with an image showing FM 1-43 or FM 4-64 labeling using Adobe Photoshop CS2.

To identify whether a given FM 1-43-positive punctum was VMAT2 positive, it was necessary to examine colabeling between VMAT2 and FM 1-43. Likewise, to determine whether sites were synaptic or nonsynaptic, Map2 labeling was examined in these same images. As such, FM 1-43positive sites were defined as being VMAT2-positive and synaptic/nonsynaptic before fluorescence at these sites was quantified as described below.

Image analysis. ImagePro v6 (Media Cybernetics) was used for counting fluorescent puncta and distance measurements. FM 1-43/4-64 intensity was measured using Leica Microsystems software. To study FM 1-43/ 4-64 fluorescence changes at labeled sites, a region of interest (ROI) was drawn around each punctum. Fluorescent puncta were selected for analysis based on fluorescence loss corresponding to field stimulation. ROIs were circular ( $1 \mu \mathrm{m}$ diameter), and positioned with the center of each ROI occupied by the center of the punctum. The mean fluorescence intensity (measured in "units") within each ROI was then determined. All suitable puncta were analyzed from each experiment. This varied widely, with each experiment providing between 1 and 22 individual puncta to the final dataset. All experimental parameters were kept identical across experiments to minimize interexperimental variation in the data.

Analysis of fluorescence data. For each presynaptic terminal, a series of 85 separate fluorescence measurements were made at $5 \mathrm{~s}$ intervals ( $7 \mathrm{~min}$ total imaging). During this period $10 \mathrm{~Hz}$ stimulation mediated a loss of fluorescence. To analyze this fluorescence loss, we modeled a first order decaying exponential to fit all 85 data points (see supplemental ST2, available at www.jneurosci.org as supplemental material, Data modeling and analysis). This procedure was individually performed for each terminal. The validity of this first order fit is discussed in supplemental ST1b, available at www.jneurosci.org as supplemental material. Note that where $2 \mathrm{~Hz}$ stimulation was used, 133 data points were acquired over $11 \mathrm{~min}$. Modeling was then performed in the same way as for data acquired during $10 \mathrm{~Hz}$ stimulation.

We calculated three key parameters directly from the model curve at each presynaptic site: $F_{\mathrm{ir}}, \Delta F$ and $\tau$, as described in supplemental ST2a, available at www.jneurosci.org as supplemental material. $F_{\text {ir }}$ was calculated as the value of the slope of the tangent to the destaining curve at $t=$ 
$0 \mathrm{~s}$. The validity of using this approach to study the initial rate of destaining is discussed in supplemental ST2b, available at www.jneurosci.org as supplemental material. $\Delta F$ was calculated from the model curve as the difference between the fluorescence intensity at $t=0 \mathrm{~s}$ and $t=\infty$. Our method of calculating $\Delta F$ is not affected by residual fluorescence that is evident after destaining (see supplemental ST1c, available at www.jneurosci.org as supplemental material). $\tau$ was also calculated directly from the model curve.

For all data presented, at least 3 separate coverslips were analyzed. In general, each coverslip was only used for a single experiment, except on three occasions where a single coverslip was used for two experiments. The maximum number of presynaptic boutons included in a dataset from a single coverslip was 23 (out of a total dataset of 81 boutons, acquired across 5 separate coverslips in total).

Statistical analysis. Where we accepted the null hypothesis, we state the $p$ value for the test, and where we rejected the null hypothesis, we indicate that the $p$ value was below the level set for significance (e.g., $p<0.05$ ). The level of significance was set at $p<0.05$, except for correlation analyses, where the significance level was set at $p<0.01$. Statistical analyses were performed using SPSS Graduate Pack v.13 (SPSS) or R (R Development Core Team, 2007).

Our method for comparing the fluorescence parameters $F_{\mathrm{ir}}, \Delta F$, and $\tau$ took account of two features of the data. First, these parameters were often not normally distributed, as determined using the Shapiro-Wilk normality test. Second, each dataset consisted of groups of observations from a number of different experiments, so that the observations were clustered within experiment. The first feature suggests that a rank-based method is appropriate, while the second feature indicates the use of a method that allows for potential within-experiment correlation. We used a method that has been developed to meet these two requirements (Datta and Satten, 2005). The method is a modified version of the Wilcoxon rank-sum test that is valid for clustered data.

Similarly, the method that we used to assess equality of variances was a modified version of Levene's test. Our approach uses a robust (sandwich) variance estimator in the ANOVA on which Levene's test is based, which allows for the clustering of observations within each independent experiment, and hence addresses potential variability between experiments. This method was developed recently (Iachine et al., 2009).

Correlation analyses were performed using Spearman's $\rho$ test.

Relationship between Pr and FM dye destaining. In our studies, we analyze the kinetics of destaining of FM dyes from synaptic and nonsynaptic sites to study the heterogeneity of Pr. To achieve this we developed a rapid approach to compare relative $\operatorname{Pr}$ at different release sites, which we describe below.

To compare the SV release properties of individual synaptic terminals, we derived a mathematical relationship between FM dye destaining kinetics and Pr. We first assume that if sufficient events could be analyzed, then, for a given stimulation frequency, probability of release $\left(P_{r}\right)$ could be estimated from the frequency of SV fusion events according to the following:

$$
P_{r} \approx N
$$

where $P_{r}$ is the probability of release and $N$ is the frequency of SV fusion events (number of fusion events/time). $N$ is measured in exocytosis events/s. This relationship is based on that previously described by Murthy et al. (1997).

We next assume that the likelihood of an FM dye-labeled SV undergoing exocytosis due to electrical stimulation (and thus detectable loss of fluorescence) is dependent on the proportion of total SVs that are labeled with FM dye. Therefore: $P_{r f}=P_{r}^{*}\left(V_{f} / V_{T}\right)$, where $P_{r f}$ is the probability of an FM dye-labeled SV undergoing exocytosis, $P_{r}$ is the probability of an exocytic event at a given synapse, $V_{f}$ is the number of SVs that are fluorescently labeled with FM dye, and $V_{T}$ is the total number of recycling SVs.

Our loading protocol used a single exposure to hyperkalemic buffer for $100 \mathrm{~s}$ in the presence of FM dye, which is sufficient to label all recycling SVs in Hpc neuron terminals (Harata et al., 2001; Mozhayeva et al., 2002). We found that increasing the time of exposure to hyperkalemic buffer did not increase the fluorescent labeling of presynaptic terminals in Hpc or midbrain neurons (data not shown). Furthermore, Mozhayeva et al. (2002) found that multiple successive exposures to hyperkalemic conditions did not result in increased FM 1-43 labeling. This demonstrated that a single exposure to hyperkalemic buffer was sufficient to label all recycling SVs. The study by Harata et al. (2001) also found that the degree of presynaptic labeling was the same whether hyperkalemic buffer or $10 \mathrm{~Hz}$ electrical stimulation was used to label recycling SVs with FM 1-43. Therefore, our loading protocol labeled all recycling SVs.

Given our assumption that all SVs are labeled at the onset of electrical field stimulation (see above), then, at $t=0, V_{f}$ is very close to $V_{T}$, and $V_{f} / V_{T} \sim 1$. This leads to: $P_{r}=P_{r f}$ (at $t=0$; where $t=0$ refers to the onset of FM dye destaining).

The remainder of our derivation therefore only applies at $t=0$, i.e., the onset of destaining, when SVs within presynaptic terminals are fully labeled with FM dye. These SVs are then stimulated to undergo exocytosis and recycling by the application of electrical field stimulation. Therefore, frequency of SV fusion events is proportional to the change in fluorescence over time at the onset of FM dye destaining:

$$
N \propto\left(F_{\text {loss }} / c\right) / t,
$$

where $F_{\text {loss }}$ is fluorescence loss due to an SV fusion event (in fluorescence units), $c$ is the fluorescence intensity per single FM dye-labeled SV (in fluorescence units), and $t$ is the time period analyzed.

By inserting Equation 2 into Equation 1, we derive an equation that relates fluorescence loss and release initial probability at the onset of FM dye destaining: $P_{r} \propto\left[\left(F_{\text {loss }} / c\right) / t\right]$; i.e.,

$$
P_{r} \propto F_{\text {loss }} /\left(c^{\star} t\right) .
$$

We assume that FM dye destaining kinetics follow first order exponential decay (see supplemental ST1b, available at www.jneurosci.org as supplemental material). In our experiments, we use data modeling to derive a first-order decaying exponential curve that describes the fluorescence data at each synapse. Specifically, we use a mixed linear model to fit a first order decaying exponential curve to the data obtained at each neurotransmitter release site. We then mathematically derive the slope of the tangent to this model destaining curve at $t=0 \mathrm{~s}$ (the onset of FM dye destaining). The derivation of this tangent is described in supplemental ST2a, available at www.jneurosci.org as supplemental material, and the rationale behind using this tangent as an estimate of the rate of fluorescence loss is discussed in supplemental ST1d and ST2b, available at www. jneurosci.org as supplemental material. The slope of the tangent to the exponential at $t=0$ describes the initial rate of decay of the curve. This initial rate of decay is a measure of the initial rate of fluorescence loss at the onset of field stimulation, and can be written simply as an expression of fluorescence loss over time: $F_{\text {ir }}=-F_{\text {loss }} / t$, where $F_{\text {ir }}$ is the slope of the tangent to our first-order destaining exponential at $t=0 \mathrm{~s}$ (note that the slope value is always negative). If we substitute this expression into Equation 3, we derive an equation that relates $F_{\mathrm{ir}}$ and $P_{r}$ at the onset of FM dye destaining: $P_{r} \propto-F_{\mathrm{ir}} / c$.

Therefore, because $c$ is assumed to be a constant that does not vary between presynaptic terminals (Murthy et al., 1997; Ryan et al., 1997; Chen et al., 2008), then at the onset of FM dye destaining: $P_{r} \alpha F_{\mathrm{ir}}$.

This demonstrates the initial rate of fluorescence loss, $F_{\mathrm{ir}}$, is proportional to the Pr at a given synaptic terminal. Based on this mathematical relationship, we used $F_{\text {ir }}$ as a relative measure of Pr at synaptic terminals. It should be noted that relative $\operatorname{Pr}$ can only be compared across terminals that were all stimulated at the same rate, e.g., $10 \mathrm{~Hz}$. $F_{\text {ir }}$ values obtained at different stimulation frequencies cannot be directly compared.

At later time points during stimulation, destained SVs will reenter the recycling pool after they have undergone endocytosis, thereby decreasing the proportion of all vesicles that are stained (i.e., $V_{f} / V_{T}$ moves away from 1). In addition, at later time points $\operatorname{Pr}$ could be affected by repeated neuronal stimulation, i.e., plasticity. Therefore, we assume that the rate of fluorescence loss is only an accurate measure of relative Pr at $t=0$ (the onset of FM dye destaining). For a discussion of the importance of only examining the initial rate of fluorescence loss in studying Pr, and how our model is able to examine relative $\mathrm{Pr}$ even at stimulation rates likely to induce short-term plasticity, see supplemental ST1d, available at www. jneurosci.org as supplemental material. 

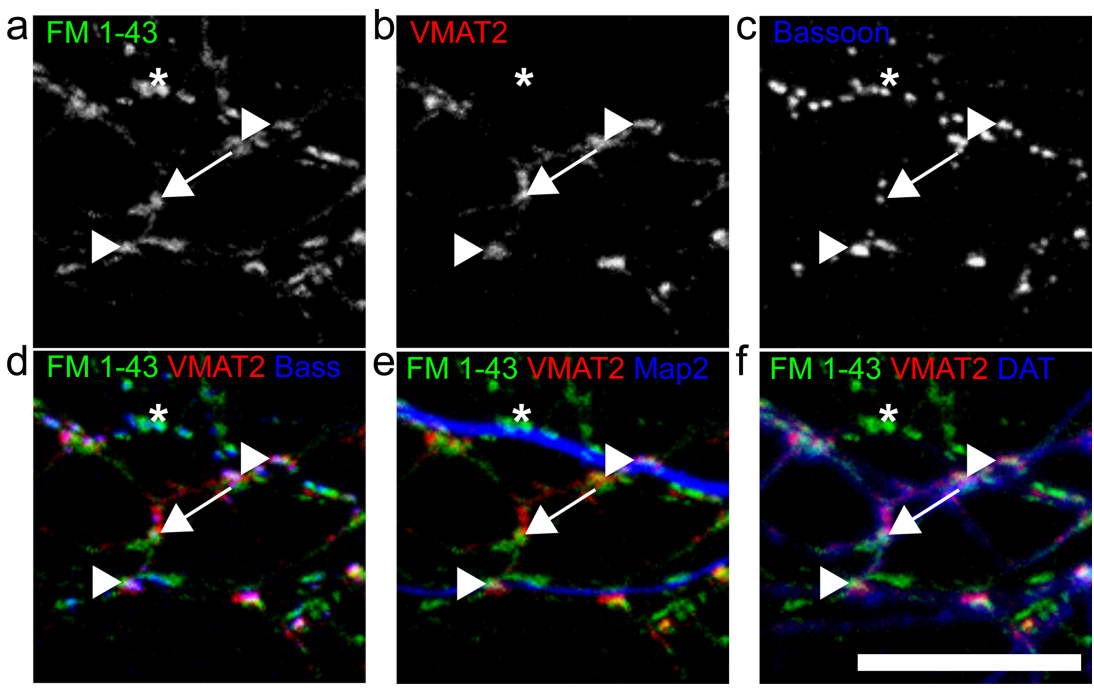

Figure 1. SV recycling at presynaptic terminals in DA neurons. Representative image of a DA neuron. $\boldsymbol{a}$, FM 1-43 was used to label recycling SVs (green in overlays). $\boldsymbol{b}$, Retrospective immunocytochemistry against VMAT2 labeled SVs in DA neurons (red in overlays). c- $\boldsymbol{f}$, Other antibodies (blue in overlays) labeled bassoon ( $(\mathbf{c}$ and $\boldsymbol{d}$ ), Map2 (e), and DAT ( $\boldsymbol{f}$ ). Most FM 1-43/NMAT2-positive sites were synaptic ( $62.0 \pm 4.4 \%$ of 659 sites, arrowheads), although nonsynaptic sites were also observed (19.3 $\pm 3.2 \%$ of 659 sites, arrow). Some FM 1-43-positive puncta did not colocalize with VMAT2 (*) and were presumably formed by non-DA neurons. Scale bar, $10 \mu \mathrm{m}$.

\section{Results}

DA neurons have synaptic and nonsynaptic sites of SV exocytosis

Styryl dyes such as FM1-43 have been extensively used to identify and analyze rapidly recycling, small neurotransmitter-containing vesicles (Murthy, 1999), which we will refer to as SVs. In this study, we were interested to use FM 1-43 to assess the functional heterogeneity of DA release sites. As our first step, we characterized the basic properties of DA release sites in DA neurons in vitro.

We stained recycling SVs in DA neurons with FM 1-43 as described in the Materials and Methods. We then imaged the FM 1-43-labeled neurons (labeling shown in Fig. 1a), and induced destaining of FM 1-43 by electrical stimulation at $10 \mathrm{~Hz}$. The neurons were subsequently fixed and labeled using an antibody directed against the vesicular monoamine transport protein VMAT2, which is localized to vesicles in DA neurons (Fig. $1 b, d-$ f). Antibodies against Map2, dopamine transporter (DAT), and bassoon were then also applied to identify dendrites, axons, and active zones in DA neurons, respectively.

We then overlaid images of the immunostained neurons on the same neuron previously labeled with FM1-43 (Fig. 1d-f). This allowed us to identify which FM1-43 sites were also VMAT2 positive. We classified VMAT2-positive sites as "synaptic" (i.e., belonging to a synapse with presynaptic and postsynaptic elements) if it was $<0.35 \mu \mathrm{m}$ from a Map2-positive dendrite [as defined by Krueger et al. (2003)]. Sites between 0.35 and $2 \mu \mathrm{m}$ from a dendrite were not analyzed. Not all VMAT2-positive sites exhibited FM 1-43 staining. The majority $(64.4 \pm 6.3 \%)$ of all VMAT2positive sites that were defined as synaptic exhibited colabeling for FM 1-43 ( $n=649$ sites, 9 experiments), demonstrating that SV recycling was apparent at most synaptic boutons of DA neurons.

We then focused specifically on VMAT2-positive sites that exhibited FM 1-43 labeling. Of all FM 1-43-labeled VMAT2positive sites, the majority $(62.0 \pm 4.4 \%)$ were synaptic $(n=659$ total FM 1-43/VMAT2-labeled sites, 13 experiments) while $19.3 \pm 3.2 \%$ were defined as nonsynaptic (located $>2 \mu \mathrm{m}$ from a dendrite). Thus, DA neurons not only possessed active synaptic terminals but also a subpopulation of nonsynaptic boutons, which contained recycling SVs and were therefore presumably capable of neurotransmitter release.

A number of regulatory proteins are localized to SVs in synaptic terminals (Sudhof, 2004). We examined the presence of these regulatory proteins at VMAT2-positive synaptic and nonsynaptic sites by immunolabeling of DA neurons. We found that VMAT2-positive sites exhibited immunolabeling for the SV proteins VAMP2 and Synaptophysin (Syp) (supplemental Fig. S1, available at www.jneurosci.org as supplemental material). While most VMAT2-positive sites colabeled with SV proteins VAMP2 $(97.3 \pm 0.5 \%)$ and Syp $(88.4 \pm 1.7 \%)$, only a subpopulation of total VMAT2positive sites colabeled for bassoon $(39.1 \pm 4.1 \%)$. These synaptic proteins are characteristically found at mature synapses in $\mathrm{Hpc}$ neurons (Krueger et al., 2003). In addition, it was evident from this analysis that not all presynaptic structures in the culture were VMAT2positive (supplemental Fig. S1, available at www.jneurosci.org as supplemental material). These VMAT2-negative sites were presumably formed by non-DA neurons, in particular GABA neurons, which are abundant in the striatum and midbrain. This is also true of sites that were FM 1-43-positive but VMAT2 negative in live cell experiments (Fig. 1).

It is notable that, although most VMAT2-positive sites did not exhibit bassoon labeling, almost all VMAT2-positive sites that exhibited FM 1-43 labeling also colabeled for bassoon. Of all synaptic sites ( $n=88$ sites from 10 experiments), $94.5 \pm 3.2 \%$ were immunopositive for bassoon. Furthermore, all FM 1-43/ VMAT2-labeled nonsynaptic sites $(n=24$ sites from 10 experiments) were also positive for bassoon. Since bassoon is a marker of active zones, these findings suggested that release of DA primarily occurs at sites that possess active zones in DA neurons.

\section{Some presynaptic DA terminals are associated with} specialized postsynaptic sites

Conventional synapses contain both a presynaptic element, formed by an axon terminal, and a specialized postsynaptic element, generally formed by a dendrite. To investigate whether any VMAT2-positive presynaptic terminals were associated with postsynaptic specializations, we next examined whether VMAT2positive puncta exhibited any colocalization with markers of postsynaptic specialization. $\mathrm{D}_{1}$ and $\mathrm{D}_{2}$ receptors are believed to be the primary target for synaptically released DA; however, there is evidence that they commonly exhibit extrasynaptic localization (Sesack et al., 1994; Yung et al., 1995). These observations suggest that D1 and D2 receptors are not robust markers of postsynaptic specialization. Therefore, to identify postsynaptic specializations, we used antibodies directed against postsynaptic proteins known to be involved in the regulation of $D_{1}$ receptor signaling: the NMDA receptor subunit NR1 and the protein PSD-95 (Harvey and Lacey, 1997; Fiorentini et al., 2003; Zhang et al., 2007). By this approach, we hoped to identify at least some of the postsynaptic sites associated with presynaptic terminals of DA neurons.

Immunolabeling against the postsynaptic proteins PSD-95 and NR1 showed that a subpopulation of VMAT2-positive sites 

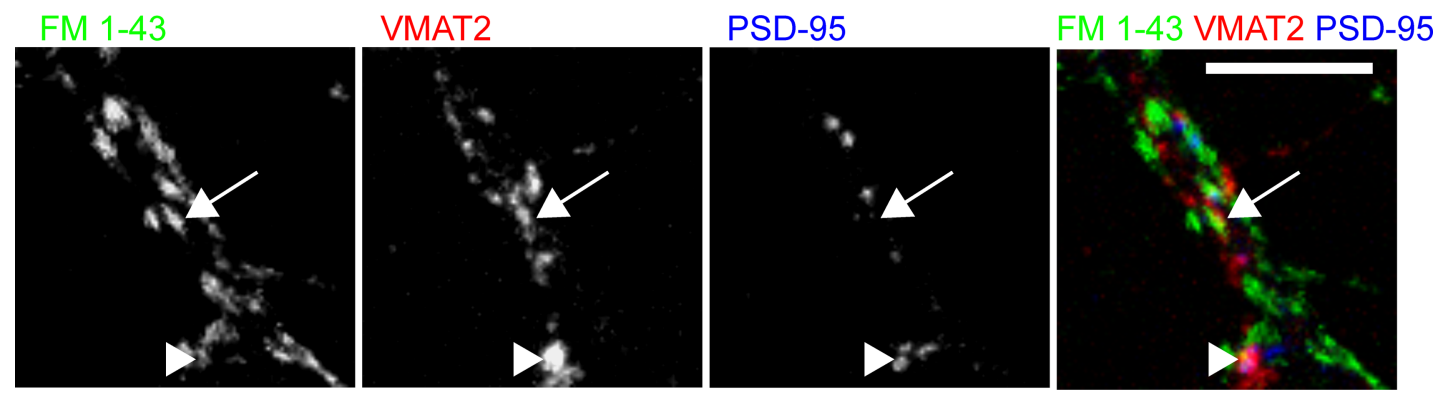

Figure 2. Association between functional presynaptic terminals of DA neurons and sites of postsynaptic specialization. A representative neuron showing labeling of FM 1-43 (green) in combination with VMAT2 (red) and PSD-95 (blue). Of all FM 1-43/VMAT2-positive sites, $46.6 \pm 2.4 \%$ exhibited colocalization with PSD-95 (arrowhead). We also observed FM 1-43/VMAT2-positive sites that did not colocalize with PSD-95 (arrow). Our observations suggested that almost half of all functional presynaptic DA terminals were associated with postsynaptic specializations. Scale bar, $10 \mu \mathrm{m}$.

exhibited colocalization against these markers $(18.9 \pm 3.3 \%$ for NR1, $n=1812$ sites from 5 neurons; $20.7 \pm 3.0 \%$ for PSD-95, $n=2186$ sites from 8 neurons). This suggested that a subpopulation of all VMAT2-positive puncta were localized to sites that had postsynaptic specializations. However, there was no indication from this data whether the VMAT2-positive sites identified were functional.

To examine whether postsynaptic specializations were present at functional VMAT2-positive sites, we next used FM 1-43 labeling and retrospective immunocytochemistry to examine the localization of PSD-95 at sites that labeled both for FM 1-43 and VMAT2-positive (Fig. 2). We found that $46.6 \pm 2.4 \%$ of all FM 1-43/VMAT2-positive sites $(n=93$ sites analyzed from 3 experiments) were colocalized with PSD-95-positive postsynaptic specializations, indicating that almost half of the functional presynaptic terminals in our culture system belonged to mature synapses with PSD-95-positive postsynaptic specializations.

Overall, our findings suggested that our DA neuron culture system contained mature presynaptic and postsynaptic structures. Based in part on these findings, we concluded that neuron cultures that were aged at least $15 \mathrm{~d}$ in vitro formed mature synapses. The reasons for using neurons of this age are further elaborated in supplemental ST1a, available at www.jneurosci.org as supplemental material.

\section{Heterogeneity of Pr in DA neurons}

Our next aim was to examine SV exocytosis at individual presynaptic sites in cultured DA neurons. To achieve this, we first labeled vesicles in midbrain neurons with FM 1-43. We next applied $10 \mathrm{~Hz}$ field stimulation to induce vesicle exocytosis, and hence FM 1-43 destaining. We opted to use $10 \mathrm{~Hz}$ stimulation for destaining as the kinetics of FM dye destaining at this frequency has been extensively studied in Hpc neurons [for examples, see the studies by Mozhayeva et al. (2002), Krueger et al. (2003), Virmani et al. (2006)]. During destaining, we acquired 85 separate measurements of average fluorescence intensity at each terminal, taking one image each $5 \mathrm{~s}$ for $7 \mathrm{~min}$. This was followed by post hoc immunostaining for VMAT2, Map2, and DAT and bassoon. We then overlaid the image of the immunostained neuron on the image of the same neuron labeled with FM 1-43. Destaining data were then only analyzed at those sites later defined as dopaminergic by virtue of the fact that there was colocalization of FM1-43 and VMAT2, in a neuron that was also immunopositive for DAT. Synaptic and nonsynaptic sites were defined as described above.

We observed that at each VMAT2-positive synaptic site that exhibited FM 1-43 labeling, fluorescence was initially lost rapidly during stimulation, after which the rate of fluorescence loss grad- ually decreased over time (Fig. 3a). The FM 1-43 destaining kinetics exhibited considerable heterogeneity across all synaptic DA terminals (Fig. 3b), suggesting that the amount of FM 1-43 destaining, and the kinetics of destaining, were highly heterogeneous across the entire population of DA terminals. Normalization of fluorescence data against $\Delta F$, a common practice in studies that use optical methods to examine presynaptic function [for examples see Mozhayeva et al. (2002); Krueger et al. (2003); Virmani et al. (2006) ], decreased the apparent heterogeneity (Fig. $3 c$ ) in the destaining profiles. Although normalization against $\Delta F$ is commonly performed, for our purposes normalization actually removed information that was important for the analysis of Pr (see Eq. 4, below).

The heterogeneity of destaining kinetics suggested heterogeneity of Pr at DA release sites. We were therefore interested to analyze the extent of the heterogeneity of $\operatorname{Pr}$ at these sites. To do this, we developed a rapid approach to compare relative $\mathrm{Pr}$ at different release sites. In our approach, we take advantage of the fact that Pr is proportional to the initial rate of fluorescence loss at a given presynaptic site (see Materials and Methods, Relationship between Pr and FM dye destaining). An example of $F_{\text {ir }}$ is shown in Figure $4 a$. Since initial rate of fluorescence loss is proportional to initial $\mathrm{Pr}$, it follows that by comparing initial rate of fluorescence loss at different neurotransmitter release sites we can gain insight into the relative initial Pr at these different sites. Simply put, a faster rate of fluorescence loss at $t=0 \mathrm{~s}$ reflects a higher initial Pr. For further discussion of the relationship between initial rate of fluorescence loss and Pr; please refer to supplemental ST1d and ST2, available at www.jneurosci.org as supplemental material.

To measure the initial rate of fluorescence loss, we assume that destaining follows first order kinetics (as discussed in supplemental ST1b, available at www.jneurosci.org as supplemental material). We then use a mixed linear model to fit a first order decaying exponential curve to the 85 separate measurements of average fluorescence intensity obtained during destaining for each release site, which provides an estimate of the entire destaining curve for a given presynaptic site. Subsequently, we mathematically derive the slope of the tangent to this model destaining curve at $t=0 \mathrm{~s}$, which we refer to as $F_{\mathrm{ir}}$, for each site (for a discussion of measuring the slope of this tangent as a measure of $F_{\text {ir }}$, see supplemental ST1d and ST2b, available at www.jneurosci. org as supplemental material). This value of $F_{\text {ir }}$ thus provides a relative measure of Pr at each FM 1-43 labeled site. It is particularly important to note that our method is only designed to examine relative $\operatorname{Pr}$ at the onset of destaining (i.e., initial $\operatorname{Pr}$, at $t=$ $0 \mathrm{~s}$ ), as discussed in supplemental ST1d, available at www. jneurosci.org as supplemental material.

Based on the above, we used $F_{\text {ir }}$ as a relative measure of $\operatorname{Pr}$ at presynaptic sites. To assess whether Pr was heterogeneous at DA 

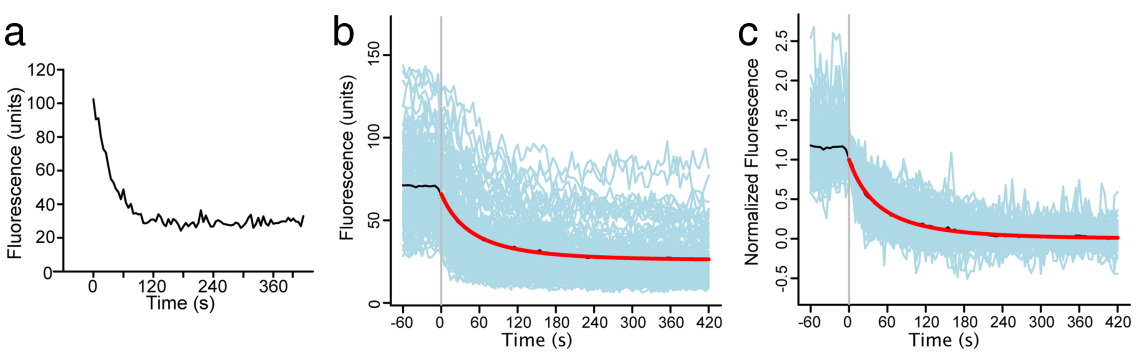

Figure 3. Average plots of fluorescence loss at synaptic sites of DA neurons. Presynaptic DA terminals stained with FM 1-43 were stimulated at $10 \mathrm{~Hz}$ to mediate destaining. Imaging commenced $60 \mathrm{~s}$ before stimulation, acquiring one image every $5 \mathrm{~s}$. Destaining commenced at $t=0$ s. $\boldsymbol{a}$, A typical trace of raw FM 1-43 fluorescence data from a single presynaptic site. Data before onset of stimulation (at $t=0$ s) is not shown. $\boldsymbol{b}$, The average plot (black) generated from all synaptic sites of DA neurons ( $n=106$ sites). A first order model of fluorescence loss, modeled to fit the average plot, is shown in red. The data plots from all 106 individual sites are shown in pale blue. Considerable heterogeneity was evident between individual synaptic sites in both neuron types. c, Normalization of fluorescence data against $\Delta F$ resulted in an apparent decrease in the degree of heterogeneity evident in the fluorescence traces.
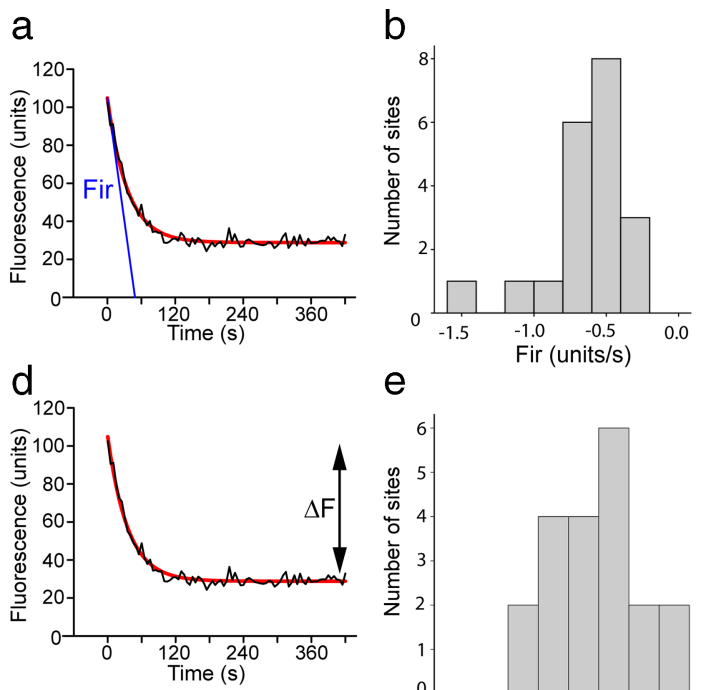

e
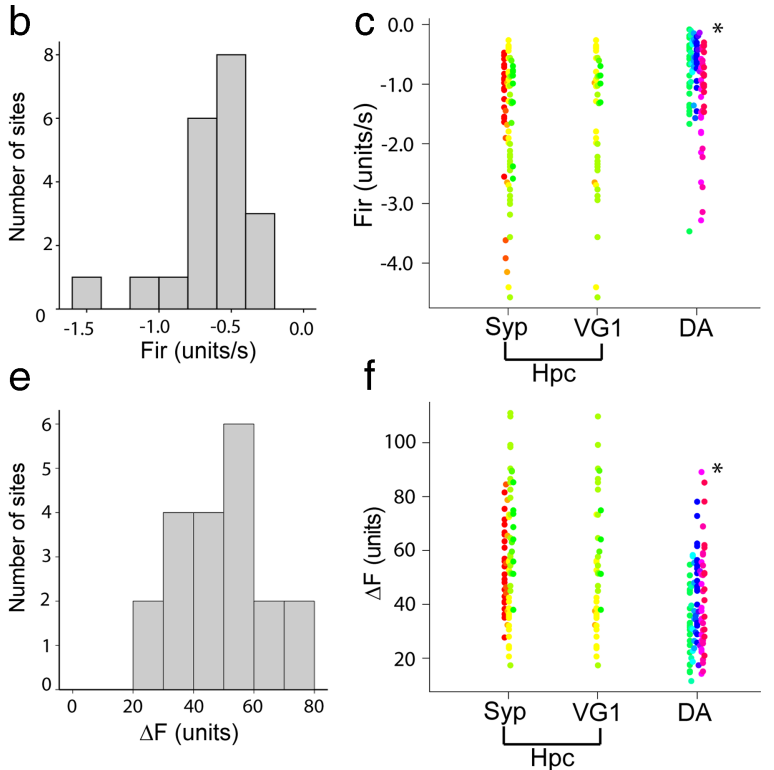

Figure 4. Synaptic terminals of DA neurons exhibit lower Pr and contain fewer recycling SVs than Hpc terminals. $\boldsymbol{a}$, Representative plot showing fluorescence loss (black) from an FM 1-43 labeled terminal in response to $10 \mathrm{~Hz}$ stimulation. A first-order exponential is fit to data (red). We mathematically derive $F_{\mathrm{ir}}$, which is a relative measure of $\mathrm{Pr}$, from the exponential curve (see supplemental ST2, available at www.jneurosci.org as supplemental material). $\boldsymbol{b}$, Frequency histogram showing $F_{\text {ir }}$ values at VMAT2-positive synaptic sites in a single representative experiment ( $n=20$ sites). $c$, Dot plots showing $F_{\text {ir }}$ data from DA synaptic sites and Hpc synaptic sites, both Syp-positive and VGLUT1-positive. Data are grouped by colors that represent independent experiments. Each dot represents an $F_{\text {ir }}$ value from a single synaptic site. $F_{\text {ir }}$ at synaptic sites of DA neurons was significantly higher $\left(^{*}\right)$ than $F_{\text {ir }}$ in Hpc neurons. $\boldsymbol{d}$, We also derived the total fluorescence loss, $\Delta F$, from the fit of the exponential curve to the raw fluorescence data. $\boldsymbol{e}$, Distribution of $\Delta F$ at VMAT2-positive synaptic sites from a single representative experiment ( $n=20$ sites). $f$, Dot plots showing $\Delta F$ values from every site analyzed, with individual experiments grouped by color as in $c$. $\Delta F$ in DA neurons was significantly lower than in Hpc neurons (*), indicating fewer recycling SVs at DA synapses.

synapses, we first examined $F_{\text {ir }}$ across many individual synaptic VMAT2-positive sites. $F_{\text {ir }}$ exhibited a broad distribution, suggesting that $F_{\text {ir }}$ was heterogeneous across synaptic sites of DA neurons ( $n=106$ total sites from 11 experiments) (Fig. $4 b$ ), and thus that Pr was heterogeneous across DA synapses.

\section{Pr at DA synapses differs from Pr at hippocampal synapses}

We next compared DA terminals with terminals of Hpc neurons. Specifically, we were interested to compare $F_{\text {ir }}$ at DA synaptic boutons with $F_{\text {ir }}$ at $\mathrm{Hpc}$ synaptic boutons, as much research has been performed previously examining $\mathrm{Pr}$ and SV release in $\mathrm{Hpc}$ neurons. Since $F_{\text {ir }}$ is proportional to $\mathrm{Pr}$, such a comparison pro- vides insight into the relative $\operatorname{Pr}$ at these two populations of synaptic terminals.

Synaptic boutons in Hpc cultures were characterized using retrospective immunolabeling with anti-Syp immunolabeling $(n=$ synaptic 81 sites from 7 experiments) (supplemental Fig. S2a, available at www.jneurosci.org as supplemental material), although to compare DA terminals specifically to glutamatergic sites we also used anti-VGLUT1 in some experiments ( $n=42$ sites from 5 experiments) (supplemental Fig. S2 $b$, available at www. jneurosci.org as supplemental material). In these experiments, most Syp-positive sites colabeled for VGLUT1 $(66.7 \pm 8.8 \%$, $n=55$ Syp-positive synaptic sites from 5 experiments analyzed) and were therefore glutamatergic. Synaptic sites were defined based on their proximity $(<0.35 \mu \mathrm{m})$ to a Map2-positive structure.

Synaptic sites of DA neurons exhibited a slower initial rate of fluorescence loss compared to Hpc synaptic sites using either Syp $(p<0.01)$ or VGLUT1 $(p<$ 0.02 ) to define Hpc terminals (Fig. $4 c$ ). This indicated that DA neurons generally exhibited lower Pr than Hpc neurons at synaptic sites.

It is well characterized that $\mathrm{Pr}$ is highly heterogeneous in synapses of Hpc neurons (Hessler et al., 1993; Rosenmund et al., 1993; Murthy et al., 1997). We were therefore interested to compare the degree of heterogeneity in Pr between DA and Hpc synapses. To examine this, we compared the variance in $F_{\text {ir }}$ in synaptic sites for these two types of neuron. We observed no significant difference in the variance of $F_{\text {ir }}$ between these two types of synapse. This suggests that Pr at DA synapses, like $\mathrm{Hpc}$ synapses, is heterogeneous, varying from synapse to synapse.

\section{Independent heterogeneous} mechanisms regulate $\mathrm{Pr}$ in DA neurons We next examined factors underlying $\mathrm{Pr}$ at DA terminals. In $\mathrm{Hpc}$ neurons, $\mathrm{Pr}$ is proportional to the number of SVs in the readily releasable pool (Dobrunz and Stevens, 1997; Murthy and Stevens, 1999; Murthy et al., 2001; Waters and Smith, 2002), which is in turn proportional to the total number of recycling SVs, called the recycling pool (Murthy and Stevens, 1999). Thus, the size of the recycling pool indirectly regulates Pr. We were therefore interested to determine if the heterogeneity of Pr we observed in DA neurons could be accounted for by variations in vesicle pool size between synapses. If so, then it would be predicted that a strong correlation would exist between $F_{\text {ir }}$ and the total vesicle pool size.

To determine whether this correlation exists, we needed to first obtain a measure of the relative recycling pool size at each neurotransmitter release site. To achieve this, we measured the total fluorescence loss during destaining $(\Delta F)$. $\Delta F$ was calculated 
from the first order model curve at each site as the difference between the fluorescence intensity at the final fluorescence intensity after destaining is complete (see supplemental ST2a, available at www.jneurosci.org as supplemental material, for calculation method), such that: $\Delta F=F_{\text {init }}-F_{\text {residual }}$, where $F_{\text {init }}=$ the initial FM 1-43 fluorescence intensity and $F_{\text {residual }}$ is the residual fluorescence observed after destaining is complete. $\Delta F$ is therefore a measure of the total recycling vesicle pool size, and is independent of variation in the residual (unreleasable) fluorescence at synaptic terminals (see supplemental ST1c, available at www. jneurosci.org as supplemental material, for further discussion regarding residual fluorescence and the calculation of $\Delta F$ ).

We investigated whether the heterogeneity of $F_{\text {ir }}$, and thus $\operatorname{Pr}$, could be explained by heterogeneity in $\Delta F$. We determined $\Delta F$ from the model curve at each synaptic DA site (shown in Fig. $4 d$, calculation method described in supplemental ST2a, available at www.jneurosci.org as supplemental material). $\Delta F$, which reflects recycling pool size, was heterogeneous at DA synaptic sites (Fig. $4 e$ ). Furthermore, the variance in $\Delta F$ was not significantly different between synaptic sites of DA and Hpc neurons, suggesting that the degree of heterogeneity in SV pool size across presynaptic terminals was similar between these two types of neuron.

When values of $\Delta F$ were compared between DA and Hpc neurons, we observed that synaptic sites of DA neurons exhibited a lower $\Delta F$ than Hpc synaptic sites (Fig. $4 f$ ), using either Syp $(p<$ $0.01)$ or VGLUT1 $(p<0.05)$ to define Hpc terminals, indicating that synaptic DA terminals contained fewer recycling SVs than $\mathrm{Hpc}$ glutamatergic terminals.

According to our decaying exponential model (see supplemental ST2a, available at www.jneurosci.org as supplemental material), $F_{\text {ir }}$ is related to $\Delta F$ by the following equation:

$$
F_{\text {ir }}=-\Delta F / \tau
$$

where $\tau$ is the time constant of fluorescence decay for a given synapse.

Equation 4 shows that $F_{\text {ir }}$ is directly related to $\Delta F$. Indeed, as predicted from this equation, there is a correlation between $F_{\text {ir }}$ and $\Delta F$ at individual synaptic DA sites (Spearman correlation coefficient, $\rho=-0.41$ ) (Fig. $5 a$ ). This relationship between $\Delta F$ and $F_{\text {ir }}$ was consistent with previous observations that recycling SV pool size was correlated with $\operatorname{Pr}$ (Murthy et al., 1997, 2001). Our findings suggest that the heterogeneity of $\operatorname{Pr}$ was at least partly due to heterogeneity of recycling SV pool size in DA neurons. We also found a correlation between $F_{\text {ir }}$ and $\Delta F$ at Hpc synaptic sites $(\rho=-0.43)$. This is consistent with previous findings in Hpc neurons (Dobrunz and Stevens, 1997; Murthy and Stevens, 1999; Murthy et al., 2001; Waters and Smith, 2002).

However, given the direct relationship between $\Delta F$ and $F_{\mathrm{ir}}$ (Eq. 4), the observed correlations were not as strong as anticipated, suggesting an additional mechanism regulating Pr. According to Equation $4, F_{\mathrm{ir}}$ is also determined by $\tau$. If $\tau$ were constant between all terminals, then $F_{\text {ir }}$ would be solely determined by $\Delta F$, with an anticipated correlation coefficient of -1 . However, if $\tau$ were variable the correlation between $\Delta F$ and $F_{\text {ir }}$ would be weaker. We therefore examined whether variation in $\tau$ accounted for the relatively weak correlations observed between $\Delta F$ and $F_{\text {ir }}$.

Values of $F_{\text {ir }}$ and $\Delta F$ from synaptic DA sites were grouped according to their value of $\tau$ (Fig. $5 b$ ). We then examined whether sites with similar $\tau$ values exhibited a stronger correlation between $\Delta F$ and $F_{\text {ir }}$ than the overall ungrouped correlation ( $\rho=$ -0.41 ). When sites were grouped according to percentiles of $\tau$, within each percentile the correlation coefficient, $\rho$, was very high, generally close to -1 (individual values shown in Fig. 5b). Therefore, the relatively weak correlation we observed between $\Delta F$ and $F_{\text {ir }}$, evident in the scatter of data in Figure $5 a$, was attributable to variation in $\tau$ across presynaptic terminals, as illustrated in Figure $5 b$. Similar results were also obtained in Hpc terminals (Fig. 5c). This observation first demonstrated the direct relationship between $\Delta F$ and $F_{\text {ir }}$, as defined by our first-order model. Second, our results showed that $\tau$ was not constant, but rather was variable across DA and Hpc terminals. According to Equation 4, the value of $\tau$ plays a direct role in determining $F_{\text {ir }}$. Therefore, our data suggest that $\tau$ reflects heterogeneous factors that contribute to Pr, and that Pr heterogeneity could not be attributed solely to variations in SV pool size.

An example of $\tau$ is shown in Figure $5 d$. When we plotted the $\tau$ values from a population of synaptic terminals of DA neurons, $\tau$ exhibited a broad, non-normal distribution with a skew toward 0 (Fig. 5e), similar to the distribution observed for $F_{\text {ir }}$. We propose that the heterogeneity of $\tau$ reflects variability of SV pool release kinetics between individual boutons (see supplemental ST1e, available at www.jneurosci.org as supplemental material).

When we examined the variance of $\tau$ in DA and Hpc synaptic boutons, we observed that there was no significant difference in the amount of variance in $\tau$ between these populations. This suggests that although the kinetics of SV pool release is heterogeneous across both DA and Hpc synapses, the degree of heterogeneity is similar in these two types of neurons. The heterogeneity of release kinetics, of which $\tau$ is a measure, has been documented previously in Hpc neurons (Waters and Smith, 2002).

Previous research has assumed a relationship between $\tau$ and $\operatorname{Pr}$ in which $\tau$ was simply determined by $\operatorname{Pr}$ (Krueger et al., 2003). According to these previous assumptions, the SV pool size would influence $\operatorname{Pr}$, which in turn would determine $\tau$. This idea makes some intuitive sense, in that synapses with higher Pr may be expected to release SVs at a faster rate, therefore exhibiting a smaller value of $\tau$.

However, the model presented by Equation 4 suggests that this previous assumption that Pr determines $\tau$ is not correct. According to Equation 4, $F_{\text {ir }}$ (which is a measure of $\mathrm{Pr}$ ) is determined by both $\Delta F$ and $\tau$. To clarify this relationship between $\tau$ and $F_{\text {ir }}$, we reasoned that if SV pool size determines $P r$, then in our model $\Delta F$ must determine $F_{\text {ir }}$. Following on, if $\Delta F$ determines $F_{\text {ir }}$, and $F_{\text {ir }}$ determines $\tau$, then $\Delta F$ must correlate with $\tau$. However, we found no significant correlation between $\Delta F$ and $\tau$ at DA terminals $(\rho=-0.161, p=0.10)$ (Fig. $5 f$ ). This demonstrated first that $\tau$ could not be determined by $F_{\text {ir }}$. Rather, this lack of correlation supported our model, in which $F_{\text {ir }}$ is determined by both $\Delta F$ and $\tau$. This suggests that $\tau$ reflects a distinct parameter that regulates Pr. Interestingly, this finding also indicates that the size of the recycling SV pool and the kinetics of SV pool release (as measured by $\tau$ ) were independent and, furthermore, that the factors reflected by $\Delta F$ and $\tau$ regulate Pr independently of each other. We will address this point further, below (see Fig. 7).

According to our model of fluorescence loss at synaptic terminals, $F_{\text {ir }}$ is determined both by the value of $\Delta F$ and the value of $\tau$ at the level of individual terminals. Having observed that values of $\Delta F$ were significantly different between DA and Hpc synapses, we next examined whether values of $\tau$ were different in these two populations of synapses. We detected no significant difference between values of $\tau$ in these two types of neuron (Fig. $5 g$ ), using either Syp or VGLUT1 to define Hpc terminals. This demonstrated that although $\tau$ is highly heterogeneous, there is no significant difference in the kinetics with which SV pool release occurs in DA and Hpc neurons.

Overall, our findings demonstrate that presynaptic terminals of DA neurons are functionally heterogeneous, exhibiting con- 

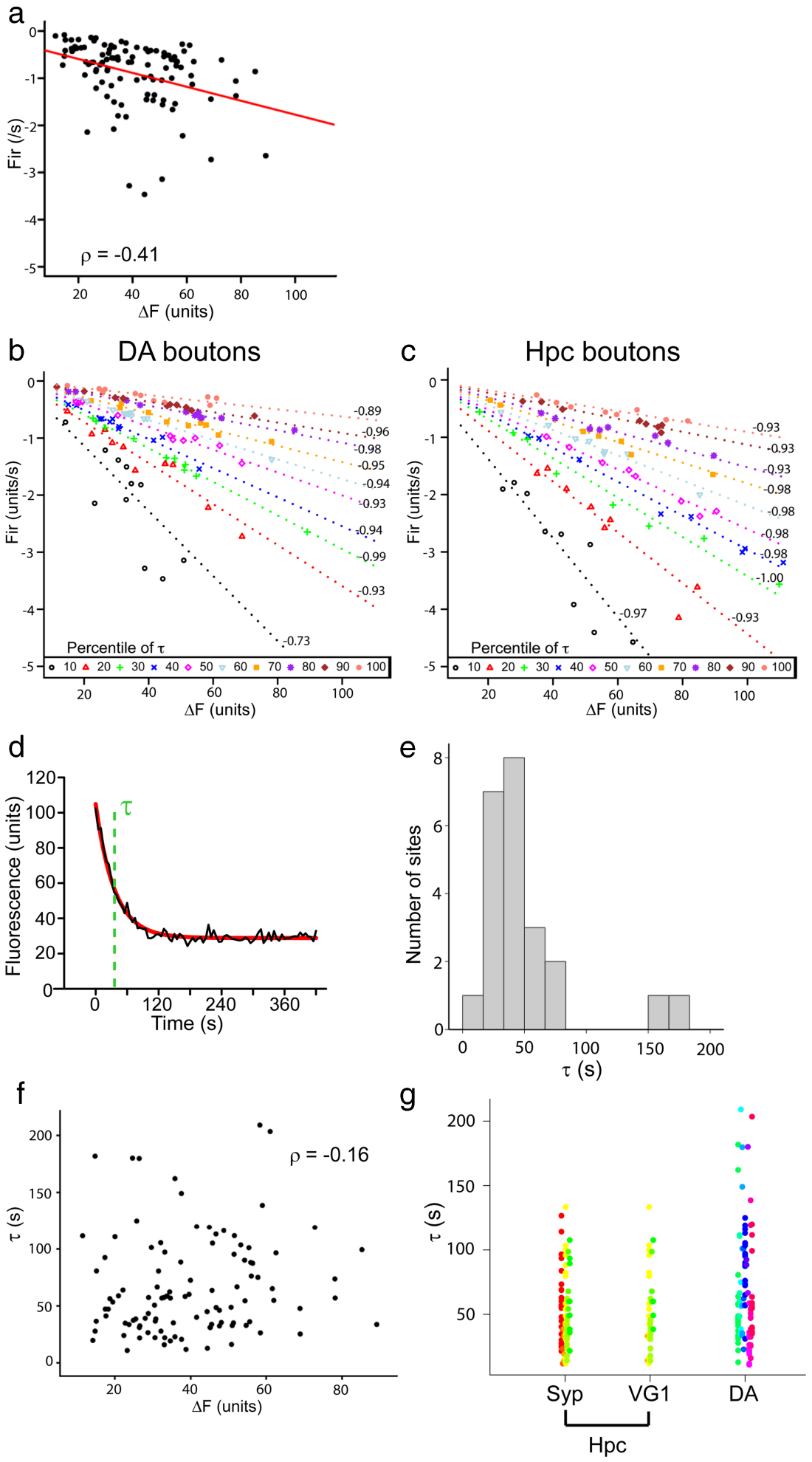

Figure 5. SV pool release kinetics is heterogeneous and independent of SV pool size. $\boldsymbol{a}$, Scatter plot of $\Delta F$ against $F_{\text {ir }}$ for all VMAT2-positive synaptic sites analyzed across all experiments ( $n=106$ sites from 11 experiments), with the linear line of best fit in red. The negative correlation between these parameters $(\rho=-0.41)$ was somewhat weaker than anticipated. To examine whether variation in $\tau$ could account for the broad spread of data when $\Delta F$ was plotted against $F_{i r}$ all synaptic sites of $D A$ neurons ( $n=106$ sites, shown in $\boldsymbol{b}$ ) and Hpc neurons ( $n=81$ sites, shown in $\boldsymbol{c}$ ) were grouped based on their percentile value of $\tau$. Percentile groups are indicated by color, with lines of best fit shown in the corresponding color. Correlation coefficients are shown adjacent. For both DA and Hpc neurons, within groups of similar $\tau, \Delta F$ was highly correlated with $F_{\mathrm{ir}}$. This observation suggested that $\tau$ was variable between individual sites. $\boldsymbol{d}, \tau$ was derived from the model exponential at each presynaptic site, as shown. $\boldsymbol{e}$, Frequency histogram showing $\tau$ at VMAT2-positive synaptic sites from a single representative experiment ( $n=22$ sites). $f$, Scatter plot showing $\tau$ against $\Delta F$ for all VMAT2-positive synaptic sites analyzed across all experiments ( $n=106$ sites from 11 experiments), with the correlation coefficient shown. No significant correlation was observed, demonstrating that SV pool release kinetics, siderable variation in $\mathrm{Pr}, \mathrm{SV}$ pool size, and SV pool release kinetics, as measured by $F_{\text {ir }}, \Delta F$, and $\tau$ respectively, at the level of individual synapses. Our findings suggested that the degree of variation was similar to that observed in Hpc neurons, in which functional heterogeneity has been well documented. In addition, DA synapses exhibit lower Pr than Hpc synapses, attributable to their lower number of recycling SVs, suggesting that DA synapses are more reluctant to release neurotransmitter than Hpc synapses.

\section{Nonsynaptic release sites are functionally similar to synaptic terminals of DA neurons}

When examining VMAT2-positive sites that exhibited FM 1-43 labeling, we observed that DA neurons formed not only synapses but also a subpopulation of nonsynaptic release sites (refer to Fig. 1). To further examine these structures, we studied FM 1-43 fluorescence loss at nonsynaptic sites in DA neurons (Fig. 6). These nonsynaptic boutons were functional structures, containing active zones and recycling SVs that label with FM 1-43, but with no apparent postsynaptic dendrite. Destaining at nonsynaptic sites exhibited first order kinetics.

We examined $F_{\text {ir }}$ at nonsynaptic boutons ( $n=31$ sites from 11 experiments) (Fig. 6). We found that the values of $F_{\text {ir }}$ were not significantly different between synaptic and nonsynaptic boutons. Therefore, there was no detectable difference between $\mathrm{Pr}$ at these two types of structure. Similar results were observed when we stimulated DA neurons at $2 \mathrm{~Hz}$ to destain synapses (see supplemental Fig. S3, available at www.jneurosci.org as supplemental material), suggesting that our observations using $10 \mathrm{~Hz}$ stimulation were not stimulation dependent (see supplemental ST1d, available at www. jneurosci.org as supplemental material). Overall, these findings suggest that synaptic and nonsynaptic sites are functionally similar in terms of their capacity for SV exocytosis.

\section{$\leftarrow$}

reflected by $\tau$, is independent of pool size at individual synaptic terminals. $g$, Dot plots showing $\tau$ at synaptic sites in DA and Hpc neurons. Data are grouped by colors that represent independent experiments. Each dot represents a $\tau$ value from a single synaptic site. No significant differences were observed in the values or variance of $\tau$ between DA and Hpc terminals, suggesting a similar degree of heterogeneity in SV pool release kinetics between these two types of neuron. 


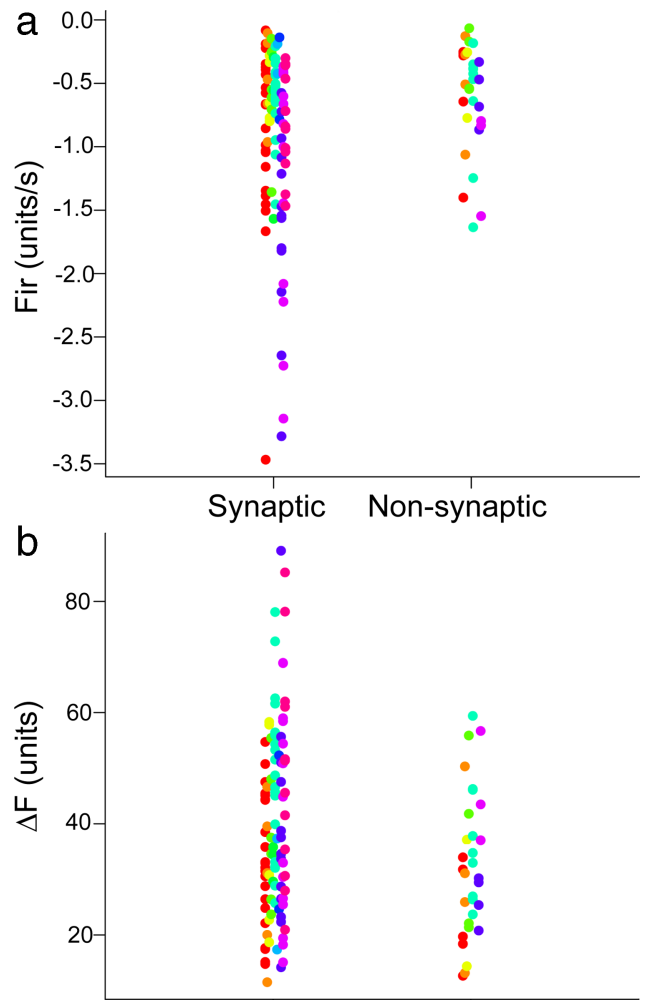

Synaptic Non-synaptic

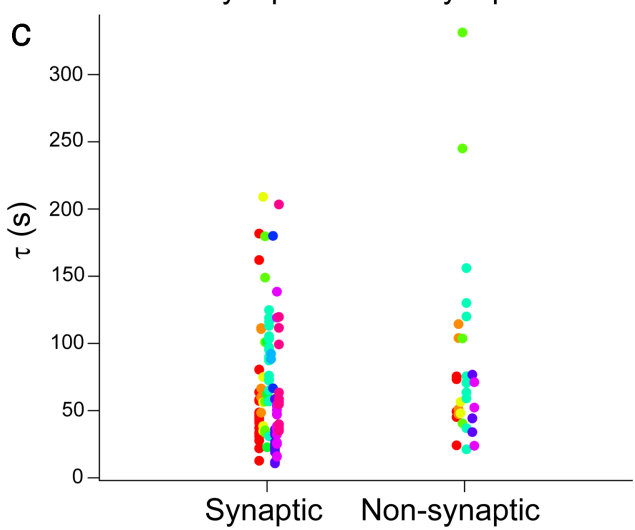

Figure 6. Nonsynaptic boutons of DA neurons are functionally similar to synaptic boutons. FM 1-43 destaining at $10 \mathrm{~Hz}$ was analyzed in VMAT2-positive sites of DA neurons and classified as either synaptic ( $n=106$ sites) or nonsynaptic ( $n=31$ sites) based on their proximity to Map2-positive dendrites. $\boldsymbol{a}$, Dot plot showing $F_{\text {ir }}$ at synaptic and nonsynaptic boutons of DA neurons. Data are grouped by colors that represent independent experiments. Each dot represents an $\tau$ value from a single VMAT2-positive site. No significant differences were observed between synaptic and nonsynaptic sites in the values of $F_{\text {ir }}, \boldsymbol{b}, \boldsymbol{c}$, We also observed no significant difference in $\Delta F(\boldsymbol{b})$ or $\tau(\boldsymbol{c})$ at synaptic and nonsynaptic boutons. These observations suggest that these two populations of boutons are functionally similar in terms of Pr, SV pool size, and SV pool release kinetics.

\section{$\tau$ can vary independently of $\Delta F$}

Decreased extracellular $\mathrm{Ca}^{2+}$ has been shown to decrease $\mathrm{Pr}$, as measured using FM 1-43, in Hpc neurons (Murthy et al., 1997). To confirm that our method of analyzing relative Pr is sensitive to conditions known to alter $\mathrm{Pr}$, we analyzed $F_{\text {ir }}$ in the presence of decreased extracellular $\mathrm{Ca}^{2+}$. When experiments were conducted in $0.5 \mathrm{mM} \mathrm{Ca}^{2+}(n=40$ sites from four experiments) instead of $2 \mathrm{mM} \mathrm{Ca}^{2+}$, we observed a significant decrease in the initial rate of fluorescence loss at synaptic sites of FM 1-43 labeling in Hpc neurons (Fig. 7a), reflecting a reduction in Pr. This finding confirms that our method is sensitive to detecting alterations in Pr.
We also observed that decreased extracellular $\mathrm{Ca}^{2+}$ resulted in an increase in the value of $\tau$ at Hpc boutons in $0.5 \mathrm{mM} \mathrm{Ca}^{2+}$ (Fig. $7 c$ ). Decreased $\mathrm{Ca}^{2+}$ did not alter $\Delta F$, however. $\Delta F$ reflects the size of the recycling pool and would not necessarily be expected to change when $\mathrm{Ca}^{2+}$ concentrations are altered.

Our data obtained using decreased extracellular $\mathrm{Ca}^{2+}$ helps to illustrate how our model of destaining works. By decreasing the $\mathrm{Ca}^{2+}$ level from 2 to $0.5 \mathrm{~mm}$, the $\tau$ value at presynaptic sites was increased. In accordance with Equation 4, this increase is $\tau$ resulted in a decrease in the initial slope of the destaining curve $\left(F_{\text {ir }}\right)$. Given that $F_{\text {ir }}$ reflects $\operatorname{Pr}$, our findings suggest that $\tau$ reflects cellular factors that regulate $\mathrm{Pr}$ and that these factors are sensitive to changes in $\mathrm{Ca}^{2+}$ concentrations. These observations also emphasize that SV pool release kinetics reflect yet-to-be-defined factors that regulate Pr independently of SV pool size.

\section{Discussion}

\section{DA neurons form synaptic and nonsynaptic boutons}

We analyzed presynaptic function in DA neurons using FM 1-43. FM 1-43 labeling was evident at $>60 \%$ of VMAT2-positive synaptic boutons. However, we observed that FM 1-43 labeling was evident at an even higher proportion of synaptic sites in $\mathrm{Hpc}$ neurons $(82.8 \pm 2.3 \%)$. This may suggest that a relatively high proportion of sites containing SVs in DA neurons are not release sites. Our findings also indicate that active zones are necessary for DA release, as they are in other neuron types (Rosenmund et al., 2003). VMAT2 colocalized with SV proteins VAMP2 and Syp, and almost half of all functional VMAT2 puncta colocalized with postsynaptic specializations, suggesting that DA neurons form mature synapses under our culture conditions (see supplemental ST1a, available at www.jneurosci.org as supplemental material).

DA axons possessed nonsynaptic boutons containing recycling SVs and active zones. Synaptic and nonsynaptic boutons exhibited similar FM 1-43 destaining properties, and thus appear equally capable of DA release. Previous research in Hpc neurons suggests that although dendrites can play a role in presynaptic maturation during synaptogenesis (Ahmari et al., 2000), neurons also possess nonsynaptic sites that are functional and mobile (Krueger et al., 2003). While their physiological relevance is unknown, nonsynaptic sites in our cultured DA neurons are reminiscent of nonsynaptic varicosities in DA axons in the brain (Descarries et al., 1996), whose role in neurotransmission remains unclear. This is a highly interesting area for future investigation.

\section{Pr is heterogeneous but relatively low at DA synapses}

Our modeling and analysis of styryl dye destaining showed mathematically that the rate of fluorescence loss at $t=0 \mathrm{~s}\left(F_{\text {ir }}\right)$ is proportional to initial Pr. As long as destaining data fits a first order model, an assumption we have justified (see supplemental ST1b, available at www.jneurosci.org as supplemental material), then we can obtain a measure of $F_{\text {ir }}$ and hence of relative $\mathrm{Pr}$, by taking the slope of the tangent to the model curve at $t=0 \mathrm{~s}$. However, our approach does not exclude alternative methods of data modeling, so long as the initial rate of fluorescence loss is estimated accurately. This method is only designed to examine relative $\mathrm{Pr}$ at the onset of destaining $(t=0 \mathrm{~s})$, and it is only possible to compare $F_{\text {ir }}$ for a specified stimulation frequency. Furthermore, providing the first order fit is valid, our analysis of relative $\operatorname{Pr}$ is not affected by subsequent short-term plasticity (see supplemental ST1d, available at www.jneurosci.org as supplemental material).

By analyzing $F_{\text {ir }}$, we observed that initial Pr was heterogeneous in DA neurons, similar to previous observations of $\mathrm{Pr}$ in $\mathrm{Hpc}$ 

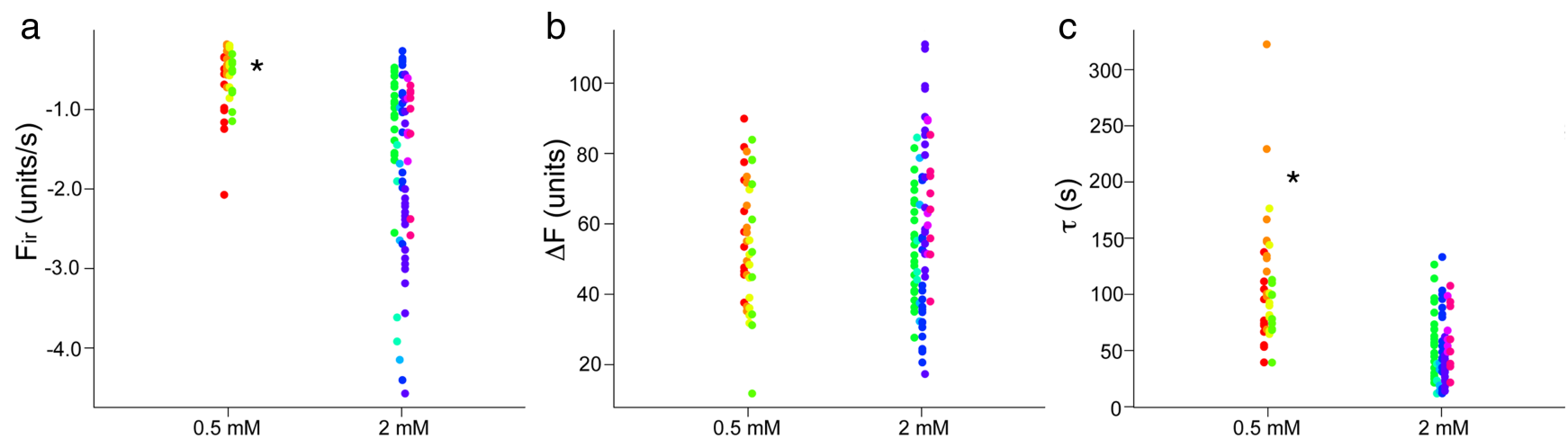

Figure 7. Decreased extracellular $\mathrm{Ca}^{2+}$ results in decreased $\tau$ and $F_{\text {ir }}$ but no change in $\Delta F$. FM 1-43 destaining at $10 \mathrm{~Hz}$ was analyzed in Syp-positive synaptic sites of cultured Hpc neurons. Cells were perfused with extracellular buffer containing either a standard ( $2 \mathrm{~mm}, n=81$ boutons from 7 experiments) or low ( $0.5 \mathrm{~mm}, n=40$ boutons from 4 experiments) concentration of $\left(\mathrm{a}^{2+}\right.$. Data are grouped by colors that represent independent experiments. Each dot represents a value from a single synaptic bouton. $\boldsymbol{a}, F_{\text {ir }}$ was significantly higher at synaptic sites stimulated in $2 \mathrm{~mm} \mathrm{Ca}^{2+}$ (right) compared with $0.5 \mathrm{~mm} \mathrm{Ca}{ }^{2+}$ (left, ${ }^{*}$ ), indicating that Pr was decreased in low $\mathrm{Ca}^{2+}$. $\boldsymbol{b}$, Decreasing $\mathrm{Ca}^{2+}$ concentration had did not alter $\Delta F$ in Hpc neurons. $\boldsymbol{c}, \tau$ was significantly lower at synaptic sites stimulated in $2 \mathrm{~mm} \mathrm{Ca}^{2+}$ (right) compared with $0.5 \mathrm{~mm} \mathrm{Ca}^{2+}$ (left, ${ }^{*}$ ). These observations showed that destaining kinetics were slowed in decreased extracellular $\mathrm{Ca}^{2+}{ }^{2}$, and that changes in $\tau$ in $0.5 \mathrm{~mm} \mathrm{Ca}^{2+}$ underlie the decrease in the initial rate of fluorescence loss.

neurons (Rosenmund et al., 1993; Dobrunz and Stevens, 1997; Ryan et al., 1997). Although Pr was heterogeneous, DA terminals generally exhibited lower Pr than Hpc terminals. Furthermore, the degree of Pr heterogeneity that we observed in Hpc neurons (coefficient of $F_{\text {ir }}$ variation $=0.66$ ) fell within the range observed in Hpc neurons by Murthy et al. (1997), suggesting that our method exhibits similar sensitivity and robustness in analyzing $\mathrm{Pr}$ heterogeneity to previous methods (see supplemental ST2b, available at www.jneurosci.org as supplemental material). Interestingly, when pooled $F_{\mathrm{ir}}$ values from DA synapses are plotted as a single histogram (supplemental Fig. S4, available at www. jneurosci.org as supplemental material), the shape of the plot, with its broad distribution and skew toward 0 , resembles the distribution of Hpc Pr published by Murthy et al. (1997).

$\Delta F$, a relative measure of recycling SV pool size, was also heterogeneous across synaptic terminals of DA neurons, similar to previous studies in Hpc neurons (Ryan et al., 1997; Moulder et al., 2007). DA terminals generally exhibited lower $\Delta F$ than $\mathrm{Hpc}$ neurons, suggesting a smaller pool of recycling SVs, to which we attribute the lower Pr at DA terminals. Previous observations in $\mathrm{Hpc}$ neurons have shown that SV pool size correlates with $\mathrm{Pr}$ (Murthy et al., 1997, 2001), which is consistent with our findings. The recycling pool in Hpc neurons is estimated between 20 and 45 SVs using FM 1-43-based techniques (Murthy et al., 1997; Murthy and Stevens, 1999; Harata et al., 2001). We infer from our data that DA terminals in vitro contain between 15 and 34 recycling SVs on average, since $\Delta F$ in DA terminals (mean $\Delta F=$ $39.97 \pm 1.62$ units) is $\sim 77 \%$ that of glutamatergic synapses (mean $\Delta F=52.33 \pm 3.57$ units). In drawing this conclusion, we assume that SVs are the same size in DA and Hpc neurons (see supplemental ST1f, available at www.jneurosci.org as supplemental material), and that large dense-core vesicles (LDCVs) do not contribute significantly to FM 1-43 loss at DA terminals (see supplemental ST1c, available at www.jneurosci.org as supplemental material).

$\tau$ was also heterogeneous at DA terminals, similar to observations at Hpc synapses (Waters and Smith, 2002). We found that in a first order model of fluorescence loss, the initial rate of fluorescence loss, $F_{\text {ir, }}$, was defined by both $\Delta F$ and $\tau$. Hence, we propose that $\tau$ measures factors regulating Pr independently of SV pool size, and that heterogeneity of $\tau$ across synapses reflects innate variability in these factors (supplemental ST1e, available at www.jneurosci.org as supplemental material). A discussion of our measurement of $\tau$ com- pared to previous values is provided in supplemental ST1, available at www.jneurosci.org as supplemental material.

We found no difference in $\tau$ between DA and Hpc synapses, demonstrating that although DA synapses contain a smaller recycling SV pool, this pool undergoes release with similar kinetics to Hpc synapses. This may suggest that there is no difference in the cellular factors that underlie $\tau$ between these neuron types.

Our findings further demonstrated that $\Delta F$ and $\tau$ are independent factors. This is shown first through the lack of correlation between these parameters, and second through our finding that $\tau$, but not $\Delta F$, was altered when extracellular $\mathrm{Ca}^{2+}$ was decreased. Waters and Smith (2002) demonstrated that phorbol ester treatment increases Pr without altering recycling pool size, which, interpreted in the context of our findings, supports our conclusion that Pr can be regulated independently of SV pool size. Furthermore, our observations suggest that the kinetics of SV release, reflected by $\tau$, are independent of the number of releasable SVs present at a presynaptic terminal.

Our method provides an approach to identify factors that regulate Pr independently of vesicle pool size, i.e., factors that regulate $\tau$. At this stage, the factors underlying $\tau$ are somewhat unclear. We show that $\tau$ is influenced by extracellular $\mathrm{Ca}^{2+}$, and that this change in $\tau$ in turn alters $F_{\mathrm{ir}}$. This suggests that $\tau$, in part, reflects molecular factors that are sensitive to $\mathrm{Ca}^{2+}$ and regulate $\mathrm{Pr}$. This could include $\mathrm{Ca}^{2+}$-sensitive molecules such as synaptotagmin I (Fernández-Chacón et al., 2001) and synapsin I (Chi et al., 2003). In Hpc neurons, it is unlikely that $\tau$ reflects the probability of SV fusion $(\mathrm{Pv})$, since in these neurons $\mathrm{Pv}$ is thought to be a constant (see supplemental ST1 h, available at www. jneurosci.org as supplemental material). However, it is unknown whether $\mathrm{Pv}$ is variable across DA synapses. Thus, in DA neurons $\mathrm{Pv}$ may contribute to variation in $\tau$, and hence to variation in $\mathrm{Pr}$. It has also been suggested that $\tau$ reflects the proportion of SVs localized in the RRP (Waters and Smith, 2002).

Plasticity occurs at $\mathrm{Hpc}$ synapses during $10 \mathrm{~Hz}$ stimulation, the predominant form of which is depletion, in which Pr declines as the RRP becomes depleted of SVs (Dobrunz and Stevens, 1997). A recent study suggested that synapses that exhibit greater depletion at $10 \mathrm{~Hz}$ stimulation subsequently exhibit slower FM dye destaining kinetics (Virmani et al., 2006), suggesting that $\tau$ may incorporate a factor defining plasticity during intense stimulation (discussed in supplemental ST1d, available at www. jneurosci.org as supplemental material). 
GABA terminals exhibit less heterogeneity in SV pool size when compared against glutamatergic terminals (Moulder et al., 2007). The authors suggested that GABA terminals may therefore be less susceptible to synaptic plasticity. When viewed in the context of the conclusions of Moulder et al. (2007), our findings that the degree of functional heterogeneity was similar between DA and $\mathrm{Hpc}$ neurons may suggest that these neuron types are similarly susceptible to plasticity. However, $\mathrm{Hpc}$ terminals with relatively low Pr tend to undergo greater short-term facilitation in response to paired-pulse stimulation (Murthy et al., 1997). Assuming that this phenomenon holds true at DA terminals, then DA synapses, which have relatively low $\mathrm{Pr}$ compared to $\mathrm{Hpc}$ synapses, may be relatively susceptible to short term facilitation. DA synapses may also be susceptible to RRP depletion, resulting in a decrease in neurotransmitter secretion during intense stimulation, assuming that $\mathrm{Pr}$ and rate of depletion are correlated in DA neurons, as has been shown at Hpc synapses (Dobrunz and Stevens, 1997). Thus, further investigation is necessary to examine these possibilities.

In conclusion, we describe a new approach to the study of relative Pr across a population of presynaptic terminals. We find that DA synapses are functionally heterogeneous. We describe previously uncharacterized differences in the regulation of SV exocytosis between DA neurons and glutamatergic Hpc neurons, while in contrast synaptic and nonsynaptic boutons in DA neurons are not different.

Drugs acting at DA synapses are used to treat neuropathologies such as PD and schizophrenia. There is also emerging evidence that synaptic function itself may be altered in PD (Serulle et al., 2007). Our approach to studying DA synapses allows further examination of signaling mechanisms that regulate DA secretion, the role of presynaptic function in disease, and examination of the effects of therapeutic drugs on synaptic function.

\section{References}

Ahmari SE, Buchanan J, Smith SJ (2000) Assembly of presynaptic active zones from cytoplasmic transport packets. Nat Neurosci 3:445-451.

Bekkers JM, Stevens CF (1989) NMDA and non-NMDA receptors are colocalized at individual excitatory synapses in cultured rat hippocampus. Nature 341:230-233.

Chen X, Barg S, Almers W (2008) Release of the styryl dyes from single synaptic vesicles in hippocampal neurons. J Neurosci 28:1894-1903.

Chi P, Greengard P, Ryan TA (2003) Synaptic vesicle mobilization is regulated by distinct synapsin I phosphorylation pathways at different frequencies. Neuron 38:69-78.

Datta S, Satten G (2005) Rank-sum tests for clustered data. J Am Stat Assoc 100:908-915.

Descarries L, Watkins KC, Garcia S, Bosler O, Doucet G (1996) Dual character, asynaptic and synaptic, of the dopamine innervation in adult rat neostriatum: a quantitative autoradiographic and immunocytochemical analysis. J Comp Neurol 375:167-186.

Dobrunz LE, Stevens CF (1997) Heterogeneity of release probability, facilitation, and depletion at central synapses. Neuron 18:995-1008.

Fernández-Chacón R, Königstorfer A, Gerber SH, García J, Matos MF, Stevens CF, Brose N, Rizo J, Rosenmund C, Südhof TC (2001) Synaptotagmin I functions as a calcium regulator of release probability. Nature 410:41-49.

Fiorentini C, Gardoni F, Spano P, Di Luca M, Missale C (2003) Regulation of dopamine $\mathrm{D} 1$ receptor trafficking and desensitization by oligomerization with glutamate NMDA receptors. J Biol Chem 278:20196-20202.

Harata N, Ryan TA, Smith SJ, Buchanan J, Tsien RW (2001) Visualizing recycling synaptic vesicles in hippocampal neurons by FM 1-43 photoconversion. Proc Natl Acad Sci U S A 98:12748-12753.

Harvey J, Lacey MG (1997) A postsynaptic interaction between dopamine
D1 and NMDA receptors promotes presynaptic inhibition in the rat nucleus accumbens via adenosine release. J Neurosci 17:5271-5280.

Hessler NA, Shirke AM, Malinow R (1993) The probability of transmitter release at a mammalian central synapse. Nature 366:569-572.

Iachine I, Petersen H, Kyvik K (2009) Robust tests for the equality of variances for clustered data. J Stat Comp Sim. Advance online publication. Retrieved Feb. 13, 2009. doi:10.1080/00949650802641841.

Krueger SR, Kolar A, Fitzsimonds RM (2003) The presynaptic release apparatus is functional in the absence of dendritic contact and highly mobile within isolated axons. Neuron 40:945-957.

Moulder KL, Jiang X, Taylor AA, Shin W, Gillis KD, Mennerick S (2007) Vesicle pool heterogeneity at hippocampal glutamate and GABA synapses. J Neurosci 27:9846-9854.

Mozhayeva MG, Sara Y, Liu X, Kavalali ET (2002) Development of vesicle pools during maturation of hippocampal synapses. J Neurosci 22:654665.

Murthy VN (1999) Optical detection of synaptic vesicle exocytosis and endocytosis. Curr Opin Neurobiol 9:314-320.

Murthy VN, Stevens CF (1999) Reversal of synaptic vesicle docking at central synapses. Nat Neurosci 2:503-507.

Murthy VN, Sejnowski TJ, Stevens CF (1997) Heterogeneous release properties of visualized individual hippocampal synapses. Neuron 18: $599-612$.

Murthy VN, Schikorski T, Stevens CF, Zhu Y (2001) Inactivity produces increases in neurotransmitter release and synapse size. Neuron 32: 673-682.

Nirenberg MJ, Chan J, Liu Y, Edwards RH, Pickel VM (1997) Vesicular monoamine transporter-2: immunogold localization in striatal axons and terminals. Synapse 26:194-198.

Pothos EN, Davila V, Sulzer D (1998) Presynaptic recording of quanta from midbrain dopamine neurons and modulation of the quantal size. J Neurosci 18:4106-4118.

Rayport S, Sulzer D, Shi WX, Sawasdikosol S, Monaco J, Batson D, Rajendran G (1992) Identified postnatal mesolimbic dopamine neurons in culture: morphology and electrophysiology. J Neurosci 12:4264-4280.

R Development Core Team (2007) R: a language and environment for statistical computing. http://www.R-project.org. Vienna: R Foundation for Statistical Computing.

Rosenmund C, Clements JD, Westbrook GL (1993) Nonuniform probability of glutamate release at a hippocampal synapse. Science 262:754-757.

Rosenmund C, Rettig J, Brose N (2003) Molecular mechanisms of active zone function. Curr Opin Neurobiol 13:509-519.

Ryan TA, Reuter H, Smith SJ (1997) Optical detection of a quantal presynaptic membrane turnover. Nature 388:478-482.

Serulle Y, Morfini G, Pigino G, Moreira JE, Sugimori M, Brady ST, Llinás RR (2007) 1-Methyl-4-phenylpyridinium induces synaptic dysfunction through a pathway involving caspase and PKCdelta enzymatic activities. Proc Natl Acad Sci U S A 104:2437-2441.

Sesack SR, Aoki C, Pickel VM (1994) Ultrastructural localization of D2 receptor-like immunoreactivity in midbrain dopamine neurons and their striatal targets. J Neurosci 14:88-106.

Staal RG, Mosharov EV, Sulzer D (2004) Dopamine neurons release transmitter via a flickering fusion pore. Nat Neurosci 7:341-346.

Sudhof TC (2004) The synaptic vesicle cycle. Annu Rev Neurosci 27: 509-547.

Tokuoka H, Goda Y (2008) Activity-dependent coordination of presynaptic release probability and postsynaptic GluR2 abundance at single synapses. Proc Natl Acad Sci U S A 105:14656-14661.

Virmani T, Atasoy D, Kavalali ET (2006) Synaptic vesicle recycling adapts to chronic changes in activity. J Neurosci 26:2197-2206.

Waters J, Smith SJ (2002) Vesicle pool partitioning influences presynaptic diversity and weighting in rat hippocampal synapses. J Physiol 541: 811-823.

Yung KK, Bolam JP, Smith AD, Hersch SM, Ciliax BJ, Levey AI (1995) Immunocytochemical localization of D1 and D2 dopamine receptors in the basal ganglia of the rat: light and electron microscopy. Neuroscience 65:709-730.

Zhang J, Vinuela A, Neely MH, Hallett PJ, Grant SG, Miller GM, Isacson O, Caron MG, Yao WD (2007) Inhibition of the dopamine D1 receptor signaling by PSD-95. J Biol Chem 282:15778-15789. 